\title{
Comparison of Clinical, Radiological and Outcome Characteristics of Ischemic Strokes in Different Vascular Territories
}

\author{
Ashraf V Valappil ${ }^{\mathrm{a}, \mathrm{c}}$, Dhanya T Janardhanan ${ }^{\mathrm{a}}$, Praveenkumar Raghunath ${ }^{\mathrm{a}}$, \\ Abdulla Cherayakkat ${ }^{\mathrm{b}}$, Girija $\mathrm{AS}^{\mathrm{a}}$
}

\begin{abstract}
Background: Ischemic strokes are usually characterized by their etiology and the affected brain territory defined by the vascular supply. Strokes located within posterior circulation are considered by some as a condition with high morbidity and mortality. Recent findings suggest that stroke etiologies of posterior circulation strokes (PCS) and anterior circulation strokes (ACS) are more alike than dissimilar, suggesting that PCS deserve same investigations as ACS. The clinical and radiological characteristics and outcome between patients with PCS and ACS were compared.
\end{abstract}

Methods: Two hundred and twenty-two consecutive ischemic stroke patients with ACS and 81 patients with PCS were prospectively analysed.

Results: Patients with ACS and PCS did not differ in terms of demographic characteristics. Mean age in ACS group was 65 yrs and PCS group was 60 yrs. Prevalence of vascular risk factors like hypertension, diabetes, dyslipidemia, coronary artery disease, smoking, past history of stroke/TIA did not differ in two groups except for hypertension. The mean National Institute of Health Stroke score (NIHSS) at admission was 11 in ACS and 8 in PCS $(\mathrm{P}=0.04)$. Brain imaging revealed pathological findings more in ACS group than PCS (CT, $72 \%$ vs. $67 \%$; MRI, $90 \%$ vs. $80 \%$ ). Cardioembolic stroke was more frequently seen in ACS than PCS (11\% vs. $6 \%)$. IV thrombolysis was done in 9 patients with ACS and 3 patients with PCS. The proportion of patients with good clinical outcome (modified Rankin score 0 - 2) was similar in ACS and PCS (43\% vs. $46 \%$ respectively, $\mathrm{P}=0.46$ ).

Conclusions: Demographic characteristics, etiology and outcome

Manuscript accepted for publication August 28, 2013

\footnotetext{
${ }^{a}$ Department of Neurology, Malabar Institute of Medical Sciences, Calicut, Kerala, India

bDepartment of Internal Medicine, Malabar Institute of Medical Sciences, Calicut, Kerala, India

${ }^{c}$ Corresponding author: Ashraf V Valappil, Department of

Neurology, Malabar Institute of Medical Sciences, Govindapuram PO,

Calicut-673016, Kerala, India. Email: drvvashraf@hotmail.com
}

doi: http://dx.doi.org/10.4021/jnr229w are more alike than different in posterior circulation strokes and anterior circulation strokes.

Keywords: Ischemic stroke; Posterior circulation stroke; Anterior circulation stroke; Comparison; Prognosis

\section{Introduction}

Ischemic strokes are usually characterized by their etiology and the affected brain territory defined by the vascular supply [1]. However, there is insufficient data on the possible impact of anatomical stroke localization upon the clinical presentation, course, and outcome [2]. Stroke located within the posterior circulation are considered by some as a condition with high morbidity and mortality, since the relatively tight packaging of numerous ascending and descending tracts, as well as nuclei, within the brainstem enables even small lesions to produce very significant neurological deficits [3]. According to the New England Medical Center Posterior Circulation registry (NEMC-PCR), stroke mechanisms of anterior circulation strokes (ACS) and posterior circulation strokes (PCS) are more alike than dissimilar, suggesting that PCS deserve the same investigations as ACS [4]. The present study aims to compare the clinical, radiological and outcome characteristics of anterior circulation and posterior circulation strokes.

\section{Patients and Methods}

The present analysis include consecutive patients with a first ever acute ischemic stroke who were admitted to the stroke unit and neurology ward at Malabar Institute of medical sciences hospital, Kerala, India, from January 2010 to December 2010. Data were collected continuously and entered into prospectively maintained stroke data base. Stroke was defined according to the World Health Organization criteria as an acute focal neurological deficit with symptoms lasting longer than 24 hours [5] and corresponding findings on brain imaging. A diagnosis of ACS was based on at least two 
Table 1. Baseline Variables by Stroke Localisation

\begin{tabular}{llll}
\hline & ACS $(\mathbf{n}=\mathbf{2 2 2})$ & PCS $(\mathbf{n}=\mathbf{8 1})$ & P value \\
\hline Gender (males), n (\%) & 139 & 57 & 0.2 \\
Age(yrs) (mean) & 64 & 61 & 0.3 \\
Hypertension, n (\%) & $173(78)$ & $48(59)$ & 0.005 \\
Diabetes mellitus, n (\%) & $104(47)$ & $37(46)$ & 0.9 \\
Dyslipidaemia, n (\%) & $116(52)$ & $42(52)$ & 0.99 \\
Coronary artery disease & $71(32)$ & $19(23)$ & 0.31 \\
Smoking, n (\%) & $87(39)$ & $35(43)$ & 0.59 \\
Previous TIA/stroke & $38(17)$ & $8(10)$ & 0.3 \\
NIHSS score (mean) & 11 & 8 & 0.04 \\
\hline
\end{tabular}

out of the three features: hemiparesis (or hemisensory loss), dysphasia (or other cortical dysfunction) and homonymous hemianopia [6]. A diagnosis of PCS was based on brainstem or cerebellar signs or isolated homonymous hemianopia [6]. All patients underwent a standard aetiological work up, including blood tests (serum electrolytes, glucose, creatinine, haemoglobin, white cell and platelet counts, prothrombin time and cholesterol levels), 12 lead ECG, cranial CT and or MRI. The following ancilliary investigations were carried out at the discretion of the treating neurologist: Doppler study of neck vessels, MR angiography and Echocardiography. The severity of the neurological deficits was assessed on admission by neurologist using the National Institutes of Health Stroke Scale (NIHSS). Antithrombotic therapy and secondary prevention was performed according to the international guidelines. The modified Rankin Scale score (mRs) was obtained at the time of discharge and 3 months after the occurrence of stroke.

\section{Statistical analysis}

Categorical variables were summarised as counts (percentage) and continuous variables as means (SD). The chi-square test was performed for cross tabulation. The following baseline variables were analysed and compared between patients with ACS and PCS: gender, age, NIHSS, arterial hypertension, diabetes mellitus, previous TIA, smoking, dyslipidemia, coronary artery disease. Usage of the ancilliary investigations with their respective major findings was compared between PCS and ACS. To compare outcomes, we divided patients into two groups with favourable (mRs score 0 - 2) and non favourable (mRs score 3 - 6) outcome respectively.

\section{Results}

A total of 303 patients were diagnosed with ischemic stroke during the study period. Two hundred and twenty-two patients had ACS (73\%) and 81 had PCS (27\%). Demographic data and vascular risk factors were not statistically different between ACS and PCS except presence of hypertension and baseline NIHSS score (Table 1). The NIHSS scores were higher in ACS than in PCS (mean 11 vs. 8; $\mathrm{P}=0.04$ ).

MRI, CT and vascular imaging were performed at similar rate in ACS and PCS (Table 2). Abnormal findings were similar in both groups. There was no significant difference in the overall spectrum of aetiologies of stroke in the two groups.

Intravenous thrombolysis with recombinant tissue plasminogen activator (rtPA) was done in 9 patients with ACS and 3 patients with PCS.

\section{Outcome}

Good outcome was defined as modified rank in score of 0,1 and 2 and mRS scores of 3, 4 and 5 were considered as bad outcome. Good outcome at discharge was observed in $43 \%$ of ACS group and $46 \%$ of the PCS group. Ten patients in ACS group and 3 patients in PCS group died. At 3 months, $66 \%$ of patients in ACS and 70\% in PCS group had a good outcome.

\section{Discussion}

The present study aimed to compare clinical and demograph- 
Table 2. Use of Diagnostic Resources and Findings

\begin{tabular}{lll}
\hline & ACS & PCS \\
& 117 & 47 \\
MRI & 13 & 4 \\
MRI \& CT & 92 & 30 \\
CT & $120(90 \%)$ & $40(80 \%)$ \\
Abnormal MRI & $67(72 \%)$ & $20(67 \%)$ \\
Abnormal CT & $30(15 \%)$ & $7(17 \%)$ \\
Vessel occlusion & & \\
\hline
\end{tabular}

ic characteristics, use of diagnostic and therapeutic resources as well as outcome between patients with ACS versus PCS treated in a tertiary hospital. Patients with PCS and ACS did not differ in terms of demographic characteristics and vascular risk factors except for the presence of hypertension, which was significantly more in patients with ACS. Brain imaging revealed pathological findings more often in ACS than in PCS. NIHSS scores on admission were significantly higher in ACS than in PCS.

Previous studies have pointed out limitations of the NIHSS score when comparing the neurologic severity of PCS and ACS [7-9]. Compared with patients with ACS, patients with PCS have higher probability of unfavourable outcome with low NIHSS scores. The reason is probably that the NIHSS is weighted towards the deficits of ACS rather than PCS. Moreover, an objective comparison of stroke severity between ACS and PCS remains challenging regarding the radiological findings. A study comparing $\mathrm{CT}$ findings to the clinical diagnosis of PCS found a lower correlation than between CT findings and clinical diagnosis of ACS. So experts consider MRI imaging the examination of choice for PCS [10]. Abnormalities on MRI were more frequently seen in ACS than PCS. Firstly, regarding the abnormalities on DWI, the vast majority of stroke patients underwent MRI during the first $24 \mathrm{~h}$ of symptom onset. However, during the first 24 h of symptom onset, $20 \%$ of patients with PCS had a false negative initial DWI study. There different reasons for low sensitivity of MRI for ischemic changes in the PC, including small lesion size compared with spatial resolution of DWI echo planar imaging, low signal to noise ratio and image artefacts in the brainstem and cerebellum [11]. As a consequence, a clinicoradiological comparison of stroke severity between ACS and PCS remains difficult.

We performed cardiac investigations (transthoracic echocardiogram and ECG) in a similar rate in both ACS and PCS. This a good change from past when patients with PCS underwent lass cardiac investigations than patients with ACS. Most of the previous studies showed cardioembolic strokes less frequently in PCS than ACS $[12,13]$. The explanation might be related to vascular anatomy. Posterior fossa structures are vascularised by narrower arteries than those of anterior circulation [14]. Approximately two fifths of brain blood flow goes into each internal carotid artery and only one fifth into vertebra-basilar arteries. By chance alone, one fifth of cardiac-origin emboli should go to the posterior circulation. Using a Y-shaped bifurcation model, it was shown that large particles preferentially enter the wider bifurcation branch [15]. The interpretation of these findings was that large emboli would tend to preferentially go into common carotid, then the internal carotid and middle cerebral arteries rather than into the narrower bifurcation branches, such as the vertebral arteries. In an in vitro model of embolus microspheres encountering the circle of Willis, the embolus trajectory was dependent on embolus size and strongly favoured the middle cerebral arteries foe large emboli [16]. We also found cardioembolic stroke more frequently in ACS than PCS (11\% vs. $6 \%$ ).

The 3 month outcome was favourable in $66 \%$ of ACS and $70 \%$ of PCS patients, and mortality was $9 \%$ in ACS and $7.5 \%$ in PCS. Even though the difference is not significant, patients with PCS had a better outcome compared to ACS. These findings demystify PCS as having a negative outcome, and goes along the relatively low mortality in patients with PCS, as reported in the NEMC-PCR [17]. Many other studies also reported a low rate of mortality and major disability in patients with posterior circulation strokes [18, 19]. Possible collateral circulation [20] and relative high threshold to ischemic damage [21] might explain in part the relative 
resistance of posterior fossa tissue to ischemia. This explains the recent data showing that time window to open occlusions in the vertebrobasilar system might be longer than that in anterior circulation [22].

In summary this study confirms that the baseline variables and risk factors of strokes in PCS and ACS are more alike than different. Moreover, the present study further demystifies the adverse prognosis of PCS, showing almost similar outcome compared with ACS.

\section{Competing Interest}

None declared.

\section{References}

1. Ng YS, Stein J, Ning M, Black-Schaffer RM. Comparison of clinical characteristics and functional outcomes of ischemic stroke in different vascular territories. Stroke. 2007;38(8):2309-2314.

2. Pinto AN, Melo TP, Lourenco ME, Leandro MJ, Brazio A, Carvalho L, Franco AS, et al. Can a clinical classification of stroke predict complications and treatments during hospitalization? Cerebrovasc Dis. 1998;8(4):204209.

3. Tao WD, Kong FY, Hao ZL, Lin S, Wang DR, Wu B, Liu M. One-year case fatality and disability after posterior circulation infarction in a Chinese hospital-based stroke study. Cerebrovasc Dis. 2010;29(4):376-381.

4. Caplan LR, Wityk RJ, Glass TA, Tapia J, Pazdera L, Chang HM, Teal P, et al. New England Medical Center Posterior Circulation registry. Ann Neurol. 2004;56(3):389-398.

5. Aho K, Harmsen P, Hatano S, Marquardsen J, Smirnov VE, Strasser T. Cerebrovascular disease in the community: results of a WHO collaborative study. Bull World Health Organ. 1980;58(1):113-130.

6. Bamford J, Sandercock P, Dennis M, Burn J, Warlow C. Classification and natural history of clinically identifiable subtypes of cerebral infarction. Lancet. 1991;337(8756):1521-1526.

7. Sato S, Toyoda K, Uehara T, Toratani N, Yokota C, Moriwaki H, Naritomi H, et al. Baseline NIH Stroke Scale Score predicting outcome in anterior and posterior circulation strokes. Neurology. 2008;70(24 Pt 2):2371-2377.

8. Libman RB, Kwiatkowski TG, Hansen MD, Clarke WR, Woolson RF, Adams HP. Differences between anterior and posterior circulation stroke in TOAST. Cerebrovasc Dis. 2001;11(4):311-316.

9. Kasner SE. Clinical interpretation and use of stroke scales. Lancet Neurol. 2006;5(7):603-612.
10. Kobayashi A, Wardlaw JM, Lindley RI, Lewis SC, Sandercock PA, Czlonkowska A. Oxfordshire community stroke project clinical stroke syndrome and appearances of tissue and vascular lesions on pretreatment ct in hyperacute ischemic stroke among the first 510 patients in the Third International Stroke Trial (IST-3). Stroke. 2009;40(3):743-748.

11. Oppenheim C, Stanescu R, Dormont D, Crozier S, Marro B, Samson Y, Rancurel G, et al. False-negative diffusion-weighted MR findings in acute ischemic stroke. AJNR Am J Neuroradiol. 2000;21(8):1434-1440.

12. Moulin T, Tatu L, Crepin-Leblond T, Chavot D, Berges S, Rumbach T. The Besancon Stroke Registry: an acute stroke registry of 2,500 consecutive patients. Eur Neurol. 1997;38(1):10-20.

13. Vemmos KN, Takis CE, Georgilis K, Zakopoulos NA, Lekakis JP, Papamichael CM, Zis VP, et al. The Athens stroke registry: results of a five-year hospital-based study. Cerebrovasc Dis. 2000;10(2):133-141.

14. Arboix A. Posterior cerebral artery infarction: an understudied vascular topography of ischaemic stroke. Eur J Neurol. 2011;18(8):1025-1026.

15. Bushi D, Grad Y, Einav S, Yodfat O, Nishri B, Tanne D. Hemodynamic evaluation of embolic trajectory in an arterial bifurcation: an in-vitro experimental model. Stroke. 2005;36(12):2696-2700.

16. Chung EM, Hague JP, Chanrion MA, Ramnarine KV, Katsogridakis E, Evans DH. Embolus trajectory through a physical replica of the major cerebral arteries. Stroke. 2010;41(4):647-652.

17. Glass TA, Hennessey PM, Pazdera L, Chang HM, Wityk RJ, Dewitt LD, Pessin MS, et al. Outcome at 30 days in the New England Medical Center Posterior Circulation Registry. Arch Neurol. 2002;59(3):369-376.

18. De Marchis GM, Kohler A, Renz N, Arnold M, Mono ML, Jung S, Fischer U, et al. Posterior versus anterior circulation strokes: comparison of clinical, radiological and outcome characteristics. J Neurol Neurosurg Psychiatry. 2011;82(1):33-37.

19. Korn-Lubetzki I, Molshatzki N, Benderly M, Steiner I. The relatively good outcome of cerebellum-brainstem ischemic strokes. Eur Neurol. 2013;69(1):8-13.

20. Liebeskind DS. Imaging the future of stroke: II. Hemorrhage. Ann Neurol. 2010;68(5):581-592.

21. Leker RR, Shohami E. Cerebral ischemia and traumadifferent etiologies yet similar mechanisms: neuroprotective opportunities. Brain Res Brain Res Rev. 2002;39(1):55-73.

22. Raphaeli G, Eichel R, Ben-Hur T, Leker RR, Cohen JE. Multimodal reperfusion therapy in patients with acute basilar artery occlusion. Neurosurgery. 2009;65(3):548552; discussion 552-543. 